\title{
Impact of Sample Preparation on Dissolution Testing: Drug Binding and Extractable Impurities and Their Effect on Dissolution Data
}

\author{
Vivek Joshi ${ }^{1,3}$, Jason Blodgett' ${ }^{1}$, Jesmi George ${ }^{1}$, and Jeff Brinker ${ }^{2}$ \\ 'Millipore Corp., Bioscience Division, 17 Cherry Hill Drive, Danvers, MA 01923 \\ ${ }^{2}$ Distek Inc., 12 North Center Drive, North Brunswick, NJ 08902
}

\begin{abstract}
Dissolution testing is a key pharmaceutical QC test required for release of oral dosage forms. It is also commonly used to predict in vivo behavior of the formulation. Filtration is a key sample preparation step that follows the drug dissolution test before sample analysis. Because of the simplicity of the filtration process, the choice of filtration devices is often ignored, leading to inaccurate and irreproducible results. This paper discusses the effects of drug binding and extractable impurities from syringe filters on dissolution data. The data presented here demonstrate that various basic and acidic drugs bind strongly to nylon membrane filters, thereby reducing drug recovery and giving an incorrect dissolution profile. On the other hand, various drugs with different physicochemical properties do not bind to hydrophilic polytetrafluoroethylene (PTFE) syringe filters, thereby leading to quantitative drug recovery. In combination with its very low extractable profile, hydrophilic PTFE is an ideal filtration choice for dissolution testing.
\end{abstract}

\section{INTRODUCTION}

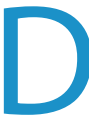
rug dissolution testing is one of the most common pharmaceutical QC tests used before approval of a manufactured drug lot. According to USP and FDA guidances $(1,2)$, dissolution testing is mandatory for all drugs when the active pharmaceutical ingredient (API) belongs to BCS Class II, III, or IV. Dissolution testing also helps predict the in vivo behavior of the oral dosage formulation. The test involves the dissolution of a solid dosage form of a drug compound under controlled conditions, followed by collection and analysis of the sample for determination of percent drug dissolved at a certain time point. This implies that following dissolution testing, filtration is a key sample preparation step to separate the dissolved drug from the undissolved dosage components. Filtration stops the dissolution process, removes undissolved drug, and allows sample analysis for amount of drug dissolved. Use of HPLC or UV-vis spectrophotometry (3) as the downstream analytical technique following dissolution also warrants filtration before analysis because particulate impurities can affect the analysis. Due to the simplicity of this sample preparation technique and the fact that sample collection and filtration are often automated, little attention is paid to this step, leading to problems downstream.

Of the various filtration formats available, syringe filters are most commonly used for sample filtration after a dissolution test. The two main issues with syringe filters that can affect the downstream analysis are analyte

\footnotetext{
${ }^{3}$ Corresponding author.
}

binding to filters and the presence of extractable impurities from the syringe filters.

Drug binding to syringe filters is well known (4) and affects downstream analysis. As the drug binds to a syringe filter, the concentration of drug in solution decreases, which affects the analysis and thereby affects the dissolution profile. Extractables, on the other hand, are soluble impurities that elute from the filter and can also affect the data.

This article presents data on the effects of analyte and membrane type on the drug binding characteristics of different syringe filters. The analysis of extractables from various syringe filters shows that pre-rinsing reduces the level of extractable impurities. In summary, this manuscript provides a rationale for choice of syringe filters following dissolution testing.

\section{MATERIALS AND METHODS}

Acetaminophen (Cat \# 1003009), ranitidine (Cat \# 1598405), loratadine (Cat \# 1370270), and ibuprofen (Cat \# 1335508) reference standards were obtained from the United States Pharmacopeia (Rockville, MD). Over-the-counter oral dosage forms of acetaminophen (Tylenol ${ }^{\circledR}$ Arthritis Relief), ranitidine (Xantac $\left.{ }^{\circledR} 75\right)$, loratadine (Claritin ${ }^{\circledR} 24 \mathrm{Hr}$ ), and ibuprofen were obtained from a local pharmacy. All other chemicals were HPLC or ACS grade and were obtained from Sigma-Aldrich (St. Louis, MO) or Fisher Scientific (Pittsburgh, PA).

Dissolution testing of drugs was carried out using the Evolution 6100 bathless dissolution apparatus (Distek, North Brunswick, NJ) following USP procedures for these formulations. Table 1 gives detailed information about the 
Table 1. Physicochemical Characteristics and Dissolution Conditions for Drugs Used in Dissolution Studies

\begin{tabular}{|c|c|c|c|c|c|}
\hline Drug & Type & $\mathbf{p K}_{\mathrm{a}}$ & Dissolution Medium* & $\begin{array}{l}\text { Time } \\
\text { (min) }\end{array}$ & $\begin{array}{l}\text { UV Analysis } \\
\text { (nm) }\end{array}$ \\
\hline Acetaminophen (Tylenol ${ }^{\circledR}$ Arthritis Pain) & Weakly acidic & 9.71 & Simulated gastric fluid without enzymes & 240 & 243 \\
\hline Loratadine (Claritin ${ }^{\circledR} 24 \mathrm{Hr}$ ) & Weakly basic & 5.0 & $0.1 \mathrm{~N} \mathrm{HCl}$ & 60 & 280 \\
\hline Ranitidine (Xantac $\left.{ }^{\circledR} 75\right)$ & Basic & 8.4 & Water & 45 & 314 \\
\hline Ibuprofen & Acidic & 4.41 & Phosphate Buffer pH 7.2 & 60 & 221 \\
\hline
\end{tabular}

${ }^{*}$ As per USP monographs

physicochemical characteristics of the analytes and their USP dissolution tests and analysis conditions. USP Apparatus 2 (paddle, 50 rpm) was used for all formulations.

\section{Choice of Dissolution Media}

Some of the most frequently used dissolution media, such as $0.1 \mathrm{~N} \mathrm{HCl}, 0.1 \mathrm{M}$ sodium acetate buffer $(\mathrm{pH} 4.5)$, and $0.2 \mathrm{M}$ sodium phosphate buffer ( $\mathrm{pH} 6.8$ ), were used for dissolution studies in addition to the media specified in the USP procedures. These dissolution media were prepared according to USP protocol. Milli-Q ${ }^{\circledR}$ water was used for all media preparation and for sample and standard dilutions.

\section{Sample Filtration}

Following the dissolution test, samples were filtered using three different Millipore syringe filters, hydrophilic PTFE (Cat \#SLCRDZ5NK), hydrophilic polyvinylidene fluoride (PVDF) (Cat \# SLHVDZ5NK), and hydrophilic nylon (Cat \#SLHNDZ5NK). Various fractions of the filtrate (first, second, third, fifth, tenth milliliter, etc.) were collected and analyzed for amount of drug dissolved using a Spectramax $384^{\circledR}$ spectrophotometer (Molecular Devices, Sunnyvale, CA). Concentration of dissolved drug was calculated using appropriate calibration curves.

\section{Analysis of Syringe Filter Extractables}

Extractable analyses were carried out using the three dissolution media mentioned earlier. A syringe filter attached to a glass syringe was filled with the extraction solvent, and after $5 \mathrm{~min}$, the solution was filtered and the first and second milliliter fractions were collected. These fractions were analyzed for the presence of extractables by reversed-phase HPLC using Waters $\mu$ Bondapak ${ }^{\mathrm{TM}} \mathrm{C} 18$ column $(3.9 \times 300 \mathrm{~mm}, 10 \mu \mathrm{m}$, USP Class L1).Water and acetonitrile were used as mobile phase components, and a gradient of $0-100 \%$ acetonitrile was used for elution of extractable impurities. A UV detector set at $214 \mathrm{~nm}$ and $254 \mathrm{~nm}$ was used to determine the presence of impurities.

\section{RESULTS AND DISCUSSION}

The choice of dissolution medium and deaeration of the medium (5), dissolution method, and sample preparation (filtration) affect data accuracy and reproducibility, which leads to an inaccurate estimation of percent drug dissolved and alters the dissolution profile. The choice of dissolution method is usually dictated by the dosage form. Careful evaluation of multiple dissolution media and media preparation strategies helps to establish the final validated dissolution medium for the test. However, limited attention is paid to sample preparation, and most often, filters available in-house are selected for the dissolution test. This can impact data if the syringe filter shows drug binding characteristics, which reduces analyte recovery, or contributes extractable impurities to the sample $(6,7)$. Inclusion of a filter bias study during method validation helps in identifying some of these problems before the method is transferred to QC labs (8).

\section{Analyte Binding}

Analyte binding to a filtration device is dependent on analyte concentration (9) and the physicochemical nature of the analyte and the filter membrane. Syringe filters have limited surface area and available binding sites, and therefore, once the membrane is saturated with the analyte, drug binding diminishes significantly and quantitative drug recovery can be obtained. Figure 1 clearly shows a drug binding effect on the dissolution of acetaminophen followed by filtration through nylon or hydrophilic PTFE syringe filters. Similarly, Figure 2 shows a drug binding effect on the dissolution of loratadine. At each time point, the first, second, and tenth milliliter of filtrate was collected and analyzed for acetaminophen concentration using spectrophotometry. When samples were filtered using a nylon syringe filter after the dissolution test, a clear drug binding effect was seen for the first milliliter of filtrate. As filtration continued through the same filter, this effect diminished, and the second milliliter of filtrate showed much higher drug recovery. Further filtration led to complete recovery of the analyte. When the same sample was filtered through a hydrophilic PTFE syringe filter, no drug binding was observed irrespective of the fraction of filtrate collected. Similar to Figures 1 and 2, Table 2 shows percent drug recovery data following the dissolution test for three different drugs (acetaminophen, loratadine, and ibuprofen) using the 
A

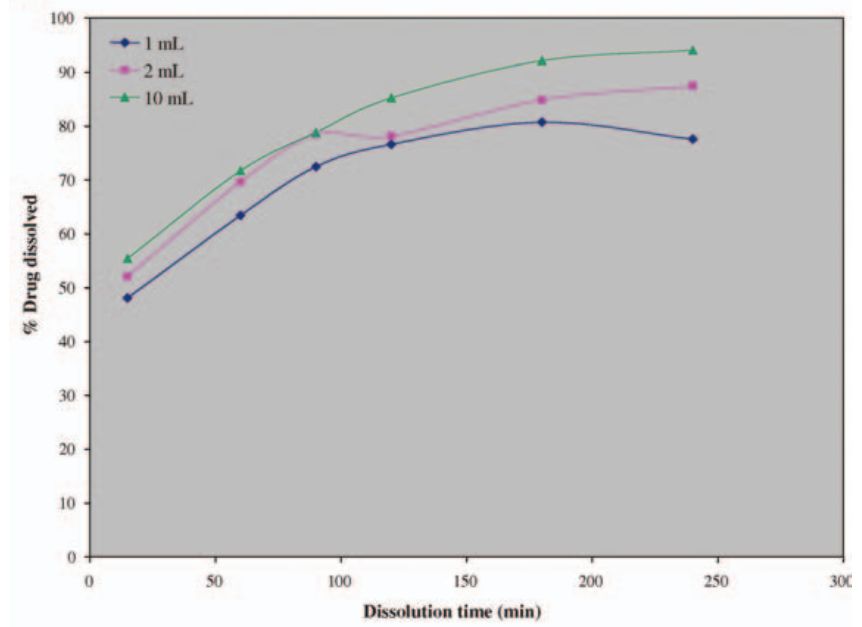

B

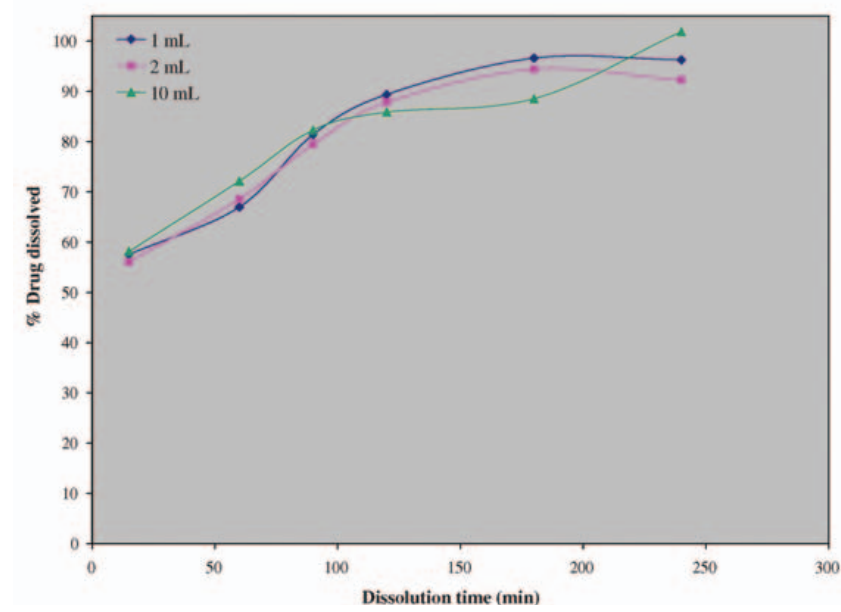

Figure 1. Effect of acetaminophen binding on recovery following filtration through (A) nylon and $(B)$ hydrophilic PTFE membrane filters.
A

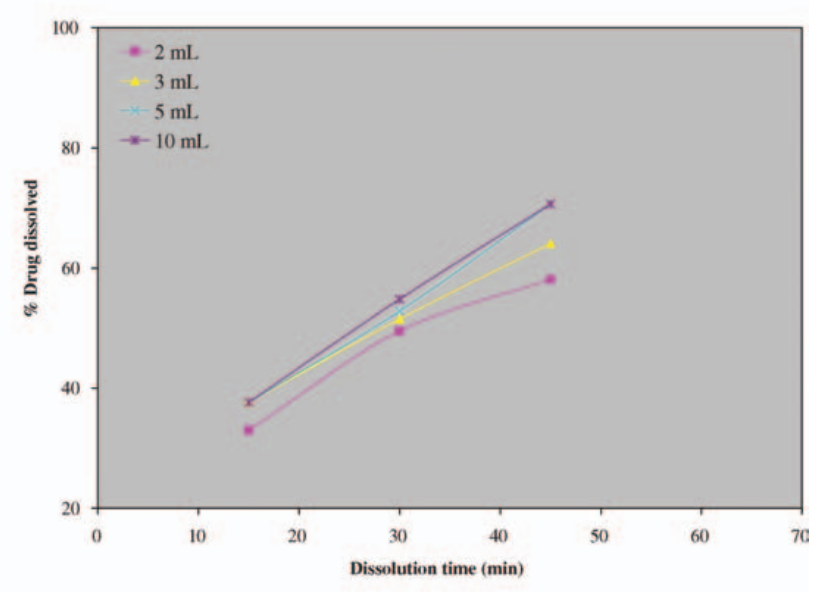

B

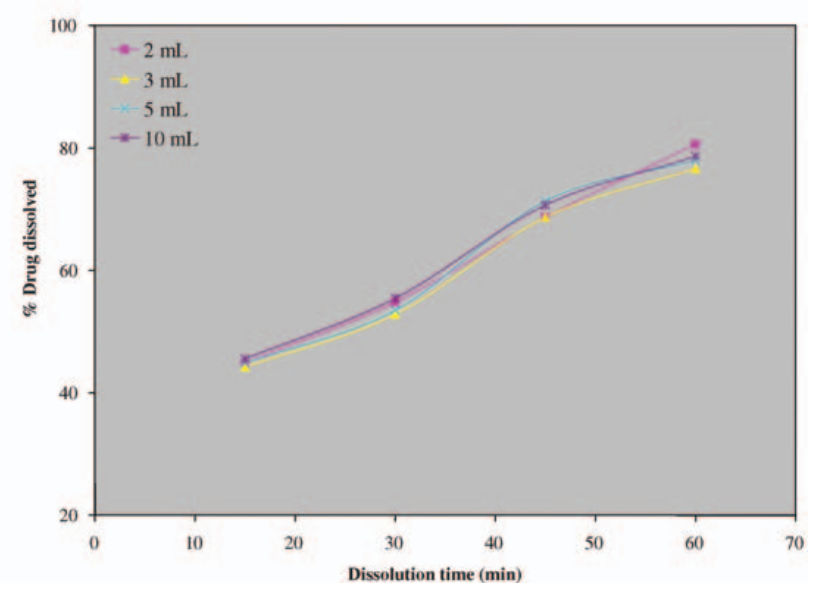

Figure 2. Effect of loratadine binding on recovery following filtration through (A) nylon and (B) hydrophilic PTFE membrane filters.

Table 2. Effect of Membrane Type on Percent Drug Recovery Following Dissolution Test and Spectrophotometric Analysis

\begin{tabular}{|c|c|c|c|c|c|c|c|c|c|}
\hline \multirow[b]{2}{*}{ Fraction collected } & \multicolumn{3}{|c|}{$\%$ Acetaminophen } & \multicolumn{3}{|c|}{ \% Ibuprofen } & \multicolumn{3}{|c|}{$\%$ Loratadine } \\
\hline & Nylon & PVDF & PTFE & Nylon & PVDF & PTFE & Nylon & PVDF & PTFE \\
\hline $1^{\text {st }} \mathrm{mL}$ & 72 & 91 & 94 & 90 & 96 & 96 & 70 & 88 & 97 \\
\hline $2^{\text {nd }} \mathrm{mL}$ & 97 & 98 & 94 & 96 & 101 & 100 & 100 & 100 & 98 \\
\hline $10^{\text {th }} \mathrm{mL}$ & 97 & 99 & 101 & 103 & 104 & 102 & 100 & 100 & 99 \\
\hline
\end{tabular}

three filters mentioned above. At the end of the dissolution test, the first, second, and tenth milliliter fractions were collected and analyzed for drug concentration using spectrophotometry. This data clearly shows reduced drug recovery when using nylon filters is a result of drug binding.
This strong drug-binding characteristic exhibited by nylon syringe filters is in line with what has been reported (4).

To evaluate whether this drug binding effect shown by Millipore nylon syringe filters is a characteristic of the particular nylon membrane used in these filters or an 
A

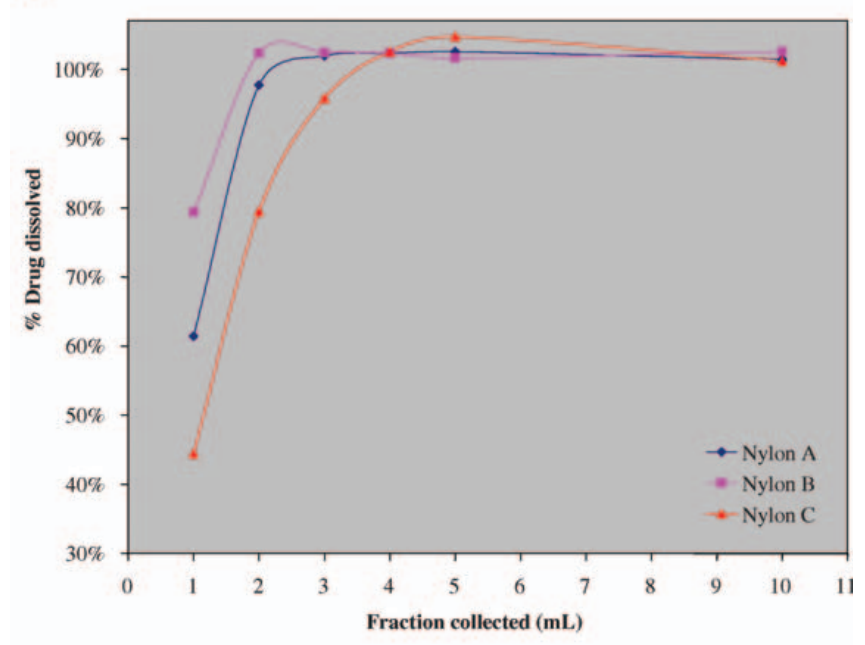

B

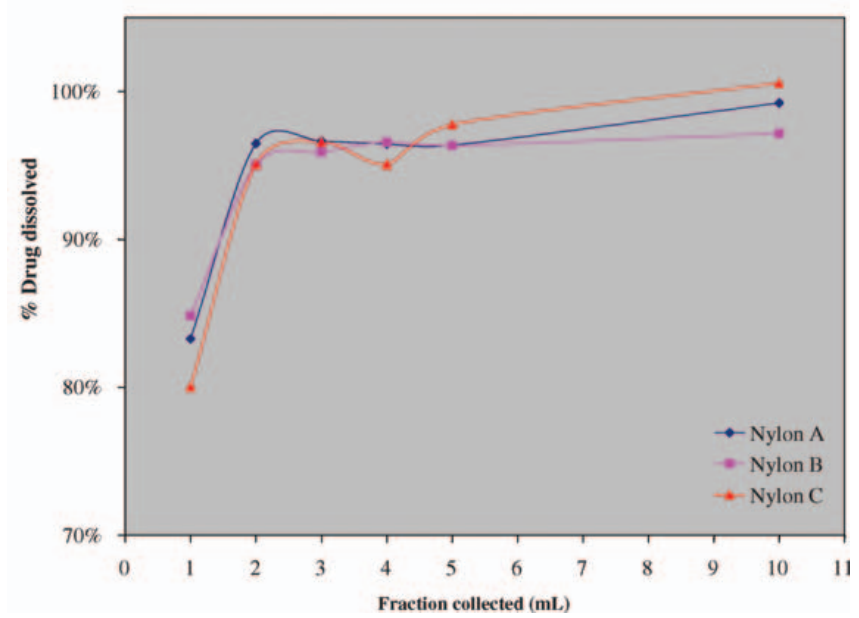

Figure 3. Binding of $(A)$ ranitidine and $(B)$ acetaminophen to nylon syringe filters from different vendors.

inherent characteristic of the nylon itself, we tested nylon syringe filters available in the market from different vendors. The effect is widespread, as can be seen in Figure 3. Nylon membrane showed strong drug binding characteristics for all the syringe filters evaluated. The percent binding is dependent on the analyte selected. Similar to ranitidine and acetaminophen, loratadine $\left(\mathrm{pK}_{\mathrm{a}}=5.0\right.$, weakly basic) also showed strong binding to nylon syringe filters (Table 2), indicating that drug binding to nylon filters is a common problem.

Drug binding to nylon can have multiple causes. Nylon membrane is inherently polar and forms hydrogen bonds with various analytes. Nylon is also capable of electrostatic interaction with different analytes depending on the $\mathrm{pH}$ of the dissolution medium. The exact mechanism was not investigated in this study since this could change from analyte to analyte. On the other hand, hydrophilic PTFE membrane, being inert, did not show strong drug binding.

\section{Extractables from Syringe Filters}

Extractables from syringe filters is another factor that can affect downstream analysis of samples (7). The presence of extractables in a syringe filter introduces impurities in the sample. Not only the syringe filter but also any surface that comes in contact with the sample has the potential to introduce extractable impurities (6). The effect of extractables on downstream analysis is not always visible. For instance, in dissolution testing followed by spectrophotometric analysis, if the extractable impurities have some absorbance at the analysis wavelength, this leads to anomalously higher analyte absorbance. On the other hand, if the dissolution test is followed by analysis by HPLC, there is a fair chance that the extractable impurity will be separated from the analyte of interest and, in that case, will be detectable.

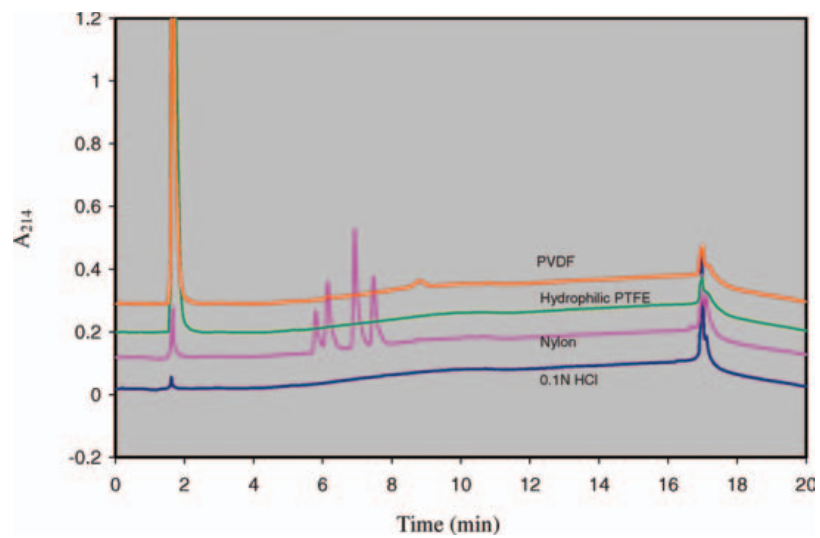

Figure 4. Extractable impurities of various syringe filters using $0.1 \mathrm{~N} \mathrm{HCl}$ as extraction solvent $(214 \mathrm{~nm})$.

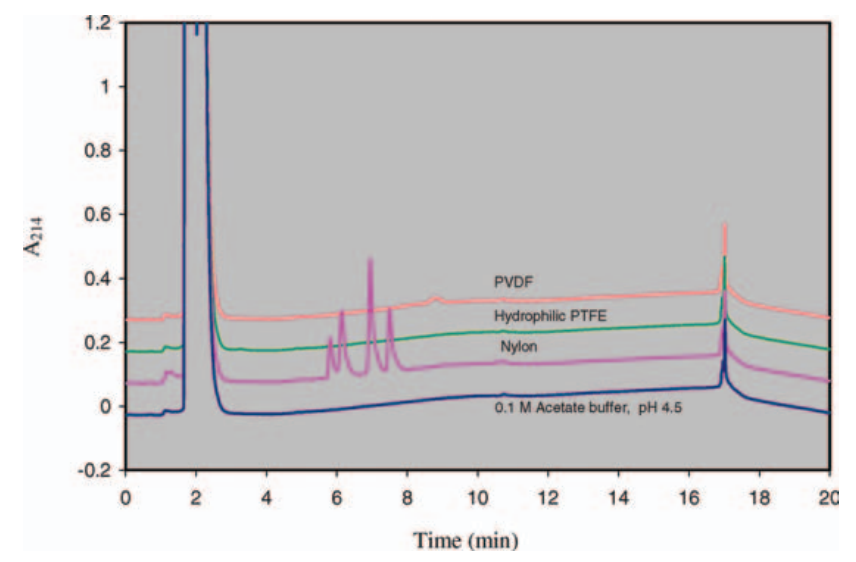

Figure 5. Extractable impurities of various syringe filters using $0.1 \mathrm{M}$ sodium acetate buffer ( $\mathrm{pH}$ 4.5) as extraction solvent (214 nm). 


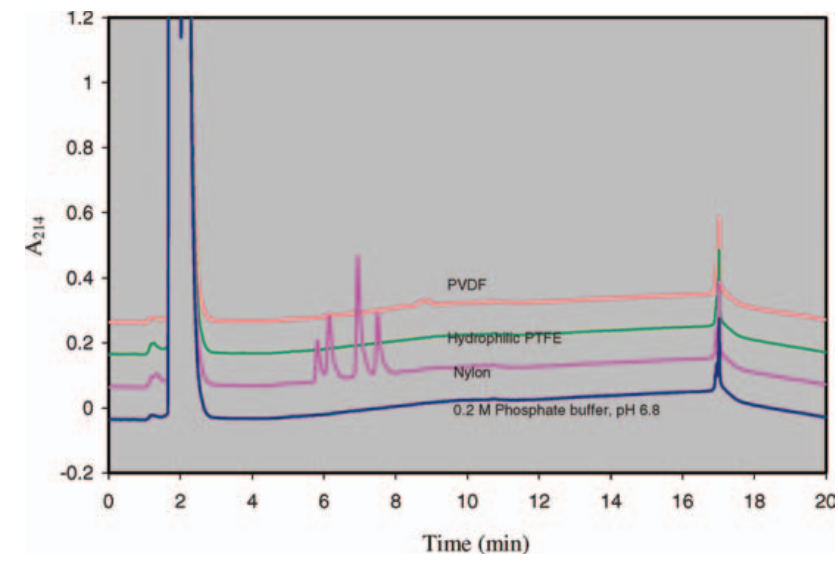

Figure 6. Extractable impurities of various syringe filters using $0.2 \mathrm{M}$ sodium phosphate buffer ( $p H 6.8$ ) as extraction solvent ( $214 \mathrm{~nm}$ ).

Figures 4-6 show the extractable profiles of nylon, hydrophilic PTFE, and hydrophilic PVDF using three commonly used dissolution media. Based on absorbance measurements at $214 \mathrm{~nm}$, these figures clearly show that hydrophilic PTFE membranes provided the cleanest sample for analysis because these filters did not introduce any extractable impurities into the sample. Nylon filters, on the other hand, had extractable impurities that were identified as cyclic low-molecular-weight polyamide oligomers (6). PVDF filters show intermediate behavior from the perspective of extractable impurities. One simple way of avoiding interference from extractables would be to use a higher wavelength for the determination of analyte concentration. Figure 7 shows that there was relatively low absorbance at higher wavelength (254 nm) following extraction using $0.1 \mathrm{~N} \mathrm{HCl}$.

Even though some of the syringe filters show extractable impurities, the level of these impurities can be

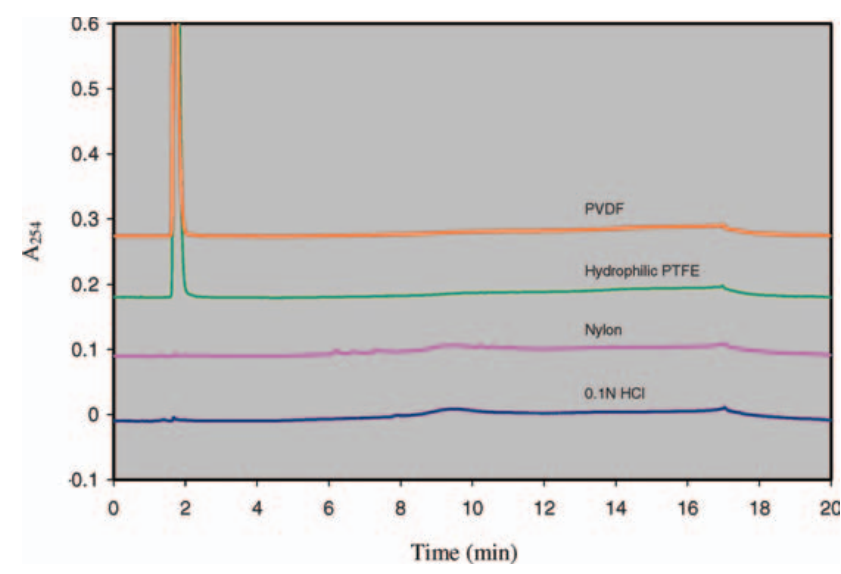

Figure 7. Extractable impurities of various syringe filters using $0.1 \mathrm{~N} \mathrm{HCl}$ as extraction solvent $(254 \mathrm{~nm})$

Dissolution Technologies | NOVEMBER 2008

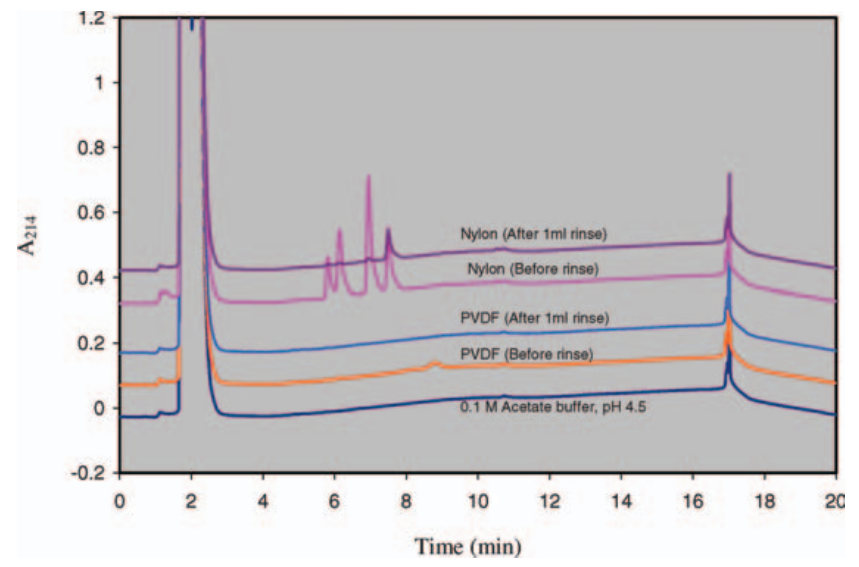

Figure 8. Effect of pre-rinsing with $0.1 \mathrm{M}$ sodium acetate buffer ( $\mathrm{pH}$ 4.5) on the level of extractable impurities from PVDF and nylon syringe filters.

reduced significantly by pre-rinsing the syringe filters with the sample. Figure 8 shows the effect of pre-rinsing with just $1 \mathrm{~mL}$ of $0.1 \mathrm{M}$ sodium acetate buffer $(\mathrm{pH} 4.5)$ on the level of extractable impurities from nylon and PVDF syringe filters, indicating that pre-rinsing would be an easy solution to alleviate extractables and drug binding.

\section{CONCLUSIONS}

In summary, drug binding and extractable impurities affect drug dissolution profiles. The data clearly show that drug binding and extractable impurities from syringe filters can affect downstream analysis significantly. Therefore, it is important to choose the right syringe filter for dissolution testing. Based on the data shown here, the lowest level of drug binding was found for hydrophilic PTFE syringe filters, followed by PVDF and nylon. In terms of the extractables profile, hydrophilic PTFE filters showed the lowest level of extractables, followed by PVDF and nylon. The data also show that pre-rinsing a syringe filter helps reduce both the drug binding and extractable level.

\section{REFERENCES}

1. Dissolution Testing of Immediate Release Solid Oral Dosage Forms; Guidance for Industry; U.S. Department of Health and Human Services, Food and Drug Administration, Center for Drug Evaluation and Research (CDER), U.S. Government Printing Office: Washington, DC, 1997.http://www.fda.gov/cder/ guidance/1713bp1.pdf (accessed Sept 29, 2008).

2. Hussain, A. S.; Lesko, L. J.; Lo, K.Y.; Shah, V.P.; Volpe, D.; Williams, R. L. The Biopharmaceutics Classification System: Highlights of FDA's Draft Guidance. Dissolution Technol. 1999, 6 (2), 5-9.

3. Wang, Q.; Ma, D.; Higgins, J. P. Analytical Method Selection for Drug Product Dissolution Testing. Dissolution Technol. 2006, 13 (3), 6-13. 
4. Lindenberg, M.; Wiegand, C.; Dressman, J. B. Comparison of the Adsorption of Several Drugs to Typical Filter Materials. Dissolution Technol. 2005, $12(1), 22-25$.

5. Nithyanandan, P.; Deng, G.; Brown, W.; Manning, R.; Wahab, S. Evaluation of the Sensitivity of USP Prednisone Tablets to Dissolved Gas in the Dissolution Medium Using USP Apparatus 2. Dissolution Technol. 2006, 13 (3), 15-18.

6. Tran, J.C.; Doucette, A. A. Cyclic Polyamide Oligomers Extracted from Nylon 66 Membrane Filter Disks as a Source of Contamination in Liquid Chromatography/ Mass Spectrometry.J. Am. Soc. Mass Spectrom. 2006, 17,652-656.

7. Pan, C.; Harmon, F.; Toscano, K.; Liu, F.; Vivilecchia, R. Strategy for identification of leachables in packaged pharmaceutical liquid formulations. J. Pharm. Biomed. Anal. 2008, 46, 520-527.

8. Skoug, J.W.; Halstead, G. W.; Theis, D. L.; Freeman, J. E.; Fagan, D. T.; Rohrs, B. R. Strategy for the development and validation of dissolution tests for solid oral dosage forms. Pharm. Tech. 1996, 20 (5), 58-72.

9. Karppi, J.; Kerman, S. A; Kerman, K. A.; Sundell, A.; K. Nyyssönen and Penttilä, I. Isolation of drugs from biological fluids by using $\mathrm{pH}$ sensitive poly (acrylic acid) grafted poly (vinylidene fluoride) polymer membrane in vitro. Eur.J. Pharm. Biopharm. 2007, 67, 562-568. 